\title{
EL CONTRATO GENERAL DE CONSTRUCCIÓN, Y EN ESPECIAL LA MODALIDAD EPC Y SUS PRINCIPALES CARACTERISTICAS
}

\author{
THE GENERAL CONSTRUCTION CONTRACT AND SPECIALLYTHE \\ EPC RULES AND REGULATIONS AND MAIN CHARACTERISTICS
}

\author{
Arturo Prado Puga
}

\section{GENERALIDADES ACERCA DEL CONTRATO DE CONSTRUCCIÓN}

\subsection{Definición}

Nuestro Código Civil configura el contrato de construcción dentro de los moldes romanos del arrendamiento de obra. $\mathrm{Y}$ a pesar de la singularidad de sus rasgos y de su poderosa autonomía, no tenemos noticias que esté prevista su tipificación en alguna legislación positiva vigente ${ }^{1}$.

En concreto, nuestro Código Civil se refiere a él, integrándolo a la disciplina del arrendamiento para la confección de una obra material (párrafo $8^{\circ} \mathrm{m}$ Tít. XXVI, art. 1915 del Código Civil) ${ }^{2}$ aunque escasamen-

\footnotetext{
"Abogado, Universidad de Chile. Doctor en Derecho, Universidad de Navarra y Profesor titular de Derecho Comercial, Universidad de Chile.

1 Sobre este tema, además de los Manuales de uso frecuente y obras de referencia general, véase en la doctrina nacional, Prat (1940); PULIdo (1962) pp. 80-109; Jerez y Palacios (1968); Huidobro (1994) pp. 20-30; Illanes (1996); Mira (2001); SAndoval (2003); Illanes (2005). En la doctrina comparada, véase MARÍa (1977) pp. 681 y ss; DEL Arco Torres y Pons (1991); García Gil (1995); García Conesa (1996); Podetti (2004); Smith, Currie \& Hancock's (2011); Bartholomew (1998) especialmente, Capítulo 2; ICE-Institution of CiviL ENGINEERS (2011) pp. 95-102.

2 Lo mismo hacen los arts. 732 y ss. del capítulo V del Código Civil de Bolivia de 1975.
}

te solo dos artículos (arts. 2003 y 2004) se refieren a la construcción de un edificio.

Tomando como idea inicial los elementos que aportan la definición del arrendamiento, se le ha definido como "aquel en que las dos partes se obligan recíprocamente la una a ejecutar una obra y la otra a pagar por ella un precio determinado" o más recientemente como "aquel en que una parte se obliga, mediante un precio convenido, a materializar en un terreno un proyecto determinado, de cualquier obra de arquitectura". Dentro de este último concepto de "obra de arquitectura", quedan englobadas tanto la construcción como las labores de refacción de la obra relativas a edificios, puentes y caminos 4 .

Cabe hacer notar, sin embargo, la tendencia actual en orden a emancipar este contrato del contrato de arrendamiento de obra, por cuanto el contrato de obra o ejecución de obra serviría para agrupar los resultados más diversos, desde la confección de un traje o una mesa hasta la construcción de una nave.

De ahí que el contrato de obra se defina en términos corrientes conforme a modelos normativos diferentes, según se trate de una obra pública o privada ${ }^{5}$. De allí

\footnotetext{
3 PRAT (1940) p. 5.

4 Jerez y Palacios (1968) p. 67.

5 García Conesa (1996) pp. 21-31.
} 
también la denominación adoptada en algunas legislaciones europeas como "contrato de empresa" (Italia), destacando la formación autónoma que le confieren algunas entidades gremiales de ese continente, al ocuparse de su estructura, funcionamiento y ejecución.

Así, por vía de ejemplo, la Confederación Nacional de la Construcción de España, ha elaborado un folleto en que define el contrato privado de ejecución de obras, canalizándolo como aquel en que "una persona denominada (constructor, contratista o empresario) se obliga a ejecutar una obra de acuerdo con un proyecto, en favor de otra (denominada propietario o promotor) y a cambio de un precio"7.

Tiene interés resaltar esta última definición, por cuanto ella se refiere al contrato privado de ejecución de obra, para distinguirlo de aquellos relativos a obras públicas donde el Estado acomete la tarea de las grandes obras de construcción de puentes, caminos, viviendas y urbanismo.

Trasladando este criterio de delimitación y estos modelos normativos a nuestro medio, el contrato privado de ejecución de obra se rige principalmente por las estipulaciones autónomas de las partes, sin alterar o vaciar el contenido de disposiciones legales imperativas vigentes, como asimismo los preceptos que supletoriamente establece el Código Civil.

El contrato de obra pública, en cambio, se entiende como aquel "inmueble

\footnotetext{
${ }^{6}$ Véase Del Arco Torres y Pons (1991) p. 19 y ss. Señalan estos autores que esta denominación también es criticada como parcial ya que "se basa en un criterio exclusivamente económico que hace referencia a grandes obras, y puede dar lugar a confusión cuando ambas partes contratantes son empresas".

7 Véase García Gil (1995)
}

construido por el Estado, directamente o en virtud de un contrato, cuya finalidad es propender al bien público"8, el cual está regido por normas administrativas que sectorialmente se han ocupado de su regulación (Obras Públicas y Viviendas), siendo el principal cuerpo que regula la materia el Decreto Supremo reglamentario $N^{\circ} 75$ del Ministerio de Obras Públicas publicado el 2 de febrero de $2004^{9}$.

En síntesis, de modo general podemos señalar que el contrato de construcción será "aquel en que una de las partes, denominada comitente o dueño de la obra, encarga a la otra, denominada contratista o constructor, la construcción de una obra determinado con relación a un plano o proyecto, obligándose a pagar por ello un precio cierto" 10 .

Cabe recordar que el art. 1996 del Código Civil señala que el contrato de construcción puede ser calificado como compraventa o arrendamiento, para lo cual distingue dependiendo de quién suministra o aporta los materiales. Si es el artífice quien lo hace el contrato es de venta y se convierte en una obra vendida presente o futura (emptio spei-emptio rei speratae); de lo contrario el contrato es arrendamiento de obra $^{11}$.

El contrato de confección de obra será de "venta" cuando es el artífice o empresario el que suministra los materiales y su perfección solo se alcanzará cuando

\footnotetext{
8 Véase Huidobro (1994) pp. 20-30.

9 Art. $4 \mathrm{~N}^{\circ} 14$ del D.S. 75. Obra Pública: Cualquier inmueble, propiedad del Estado, construido, reparado o conservado por este, en forma directa o por encargo a un tercero, cuya finalidad es propender al bien público.

10 María (1977) pp. 681-683, citado por Del Arco TORres y PONS (1991) p.21.

11 García Conesa (1996) pp. 32-37.
} 
se aprueba la obra por aquel que la ordena. En cambio será de "arrendamiento de servicios", cuando los materiales son suministrados o proporcionados al artífice o empresario, por el comitente que encarga la confección de la obra.

La importancia de esta clasificación, en ambos casos, radica en la asunción de los riesgos de pérdida de los materiales que se desplazan y se transfieren al que ordena la obra con suministro de materiales o bien al artífice o constructor dependiendo una vez que esta haya sido reconocida y aprobada (art. 2000 del CC).

\subsection{Contrato de construcción a} SUMA ALZADA O DE "AJUSTE ALZADO” O "A TANTO ALZADO"

Bajo esta modalidad, el constructor, contratista o empresario, dirige, ejecuta y administra la obra hasta su total terminación por un precio cierto, global y único aportando el trabajo y los materiales. El precio convenido con el mandante o comitente o con el propietario del terreno que encomienda la ejecución de la obra, se mantendrá invariable ${ }^{12} \mathrm{o}$ inamovible salvo

12 García Conesa (1996) pp. 146 y ss. Por su parte, Mira Ripolles colaciona la aplicación de los principios generales de la contratación aplicables a la obra pública y destaca el Dictamen D 21990/86 sobre este aspecto. "Si por defectos técnicos que hacen incongruente elaborado por la autoridad contratante y el contratista se ve obligado a utilizar un método constructivo diferente que significa la realización de una obra distinta a la programada, ya que por deficiencias técnicas del proyecto no era compatible continuarlo, tiene derecho al pago de la obra nueva o extraordinaria (concepto que se contrapone al de suma alzada) en cuanto han sido necesarias por circunstancias imprevisibles para el debido cumplimiento de lo pactado, única manera de no infringir los principios de buena fe que deben imperar en la celebración y cumplimiento de los contratos, de equilibrio económico que que se establezca una cláusula de revisión de precios o que se introduzcan obras extraordinarias o bien reformas o modificaciones introducidas en el proyecto por el comitente que impliquen un aumento o disminución de la obra, en cuyo caso la estipulación del precio inalterable pierde su fuerza obligatoria y debe ser renegociada. Por lo general, la revisión del precio dependerá del tiempo transcurrido desde el inicio de la obra y de la incidencia que pueda revestir en el monto total.

La ventaja para el comitente es manifiesta ya que conoce en forma anticipada el precio final de la obra. Por este motivo, el contratista o constructor debe prever inicialmente y en forma realista sus precios en función del riesgo que acepta asumir, y de la realidad del mercado, particularmente en períodos de inflación.

Presenta interés esta modalidad por cuanto en ella se advierte con nitidez que el contratista se obliga respecto del comitente o propietario a la obtención del resultado proyectado al que se debe encaminar toda su actividad ${ }^{13}$. Se trata de un arrendamiento de obra que podría aproximarse por sus caracteres a la compraventa de cosas futuras.

Lo esencial -para ser fieles a la conocida distinción ${ }^{14}$ entre la obligación de medio y de resultados- radica en que la obra es ejecutada por el contratista a cambio de un

deberá preservarse en todo convenio de carácter conmutativo, y de enriquecimiento sin causa”.

13 Del Arco Torres y Pons (1991) p. 23. "La doctrina es casi unánime en puntualizar que, la distinción entre estos dos contratos, la diferencia esencial entre el arrendamiento de servicios y el de obra (llamado también contrato de empresa), radica en que la prestación está constituida por el servicio en sí, mientras que en este la prestación está representada por el 'resultado', sin que importen para nada las horas de trabajo, es decir, el servicio".

14 Weil y Terré (1975) pp. 431-438. 
precio o costo cierto y en principio irrevocable, la cual debe ser aprobada y recibida conforme por el comitente al momento de su terminación.

Lo fundamental es que el riesgo económico de este contrato, de precio ajustado o cerrado, como sucede en la mayoría de los casos, se supone que es el que se considera al momento de su celebración, con las especificaciones y dificultades previsibles que entrega el mandante en ese momento pero con la ventaja que simplifica la administración del contrato, especialmente, en cuanto a los gastos generales, utilidades y a los estados de pago.

Bajo esta modalidad, toda alteración sobrevenida durante su ejecución y antes de su consumación presupone obligadamente una modificación de las condiciones del contrato original ya que como indica un autor "ambas han determinado de modo preciso y claro el quantum de las obligaciones de cada una, estableciendo una verdadera ecuación" 15 .

Lo contrario importaría, como se ha sostenido ${ }^{16}$, que la obligación que origina el contrato carece de causa o justificación.

15 Podetti (2004) p. 252, siguiendo a Mosset ITURRASPE.

16 Con respecto al estudio de la cláusula rebus sic stantibus por excesiva onerosidad sobrevenida en relación a la revisión del precio ajustado en un contrato de construcción, parece interesante colacionar la Sentencia de la Audiencia Territorial de Oviedo, España de fecha 15 de febrero de 1978 que señala: "Considerando: Que en cuanto a la aplicación de las citadas cláusulas de revisión de los contratos fundadas en circunstancias posteriores al contrato que influyen de modo sensible en el equilibrio de las prestaciones, se han elaborado por la doctrina científica diversas teorías para justificar que el principio de irrevocabilidad o inmodificación del contrato no es absoluto, teorías que basadas, bien en la presuposición de la base del negocio, en la equivalencia de las prestaciones en la propia causa del mismo, en el riesgo imprevisible, en la
El problema fundamental que se plantea entonces, bajo esta modalidad, serán las variaciones que sean una consecuencia de la ejecución del contrato y que afecten durante este transcurso el equilibrio de las obligaciones recíprocas, esto es, un cambio genético o una disociación en el precio tratado y establecido como fijo e invariable en el contrato desde su formación hasta su costo sobreviniente, originado en la variación real y liquidación final ${ }^{17}$.

Siendo este tema de relevancia suma, no podemos afirmar que exista un criterio de solución uniforme existiendo numerosas opiniones que basculan entre la fidelidad a la seguridad jurídica que brinda un contrato pactado bajo esta modalidad de precio, sin compensaciones adicionales por imprevistos por más gravosa que ella sea y, por otra parte, la invocación a los principios generales del derecho y la buena fe que exigen su adaptación en aras de restablecer el equilibrio contractual. ${ }^{18}$

buena fe y equidad, en el abuso del Derecho o en el enriquecimiento injusto, no han sido ajenas a la jurisprudencia de nuestro Tribunal Supremo...”. Sobre este aspecto véase De Amunátegui (2003) pp.123 y ss. Que recoge las diversas posiciones de la doctrina y las fórmulas o remedios arbitrados para buscar una solución. También en PodetTi (2004) pp. 212-215, con el comentario de los Códigos Argentino, Peruano y Brasileño y los Principios de UNIDROIT acerca de esta cláusula de excesiva onerosidad cuya protección y corrección se conoce en el derecho inglés como hardship.

Véase el art. 737 del Código Civil de Bolivia "Variación al Proyecto". En cambio, en países donde incluso se reconoce la teoría de la imprevisión esta posibilidad está vedada. Así por ejemplo se recoge en el Código Civil argentino el cual en su art. 1633 señala: "Aunque encarezca el valor de los materiales y de la obra de mano, el locador bajo ningún pretexto puede pedir aumento en el precio, cuando la obra ha sido contratado por una suma determinada”.

17 García Conesa (1996) p. 151.

18 En nuestro medio, además del conocido trabajo de De la Maza (1933) y la obra Abeliuk (1983), en 


\subsection{RefERENCia a los modelos NORMATIVOS DE CONTRATOS DE CONSTRUCCIÓN CONTENIDOS EN EL SISTEMA FIDIC}

El uso social extendido y global de los contratos de construcción para grandes obras y la necesidad de contar con modelos objetivos de alcance general frente a la parquedad de previsiones normativas existentes en buena parte de los países bajo régimen de codificación, suele dar lugar a que se recurra a la disciplina de los que se conocen como modelos FIDIC que emanan de la "Federación Internacional de Ingenieros Consultores" fundada en 1913, organismo que ha sido capaz de crear moldes jurídicos adaptados a los esquemas que exige una figura compleja y cambiante que cada país ha ido instalando al amparo del contrato de construcción tradicional como contrato nominado típico ${ }^{19}$, cobijados por lo tanto, bajo los límites de sus categorías legales imperativas a los que se debe ajustar y en especial, en cuanto a suplir sus deficiencias y aplicar sus normas de interpretación.

Cabe señalar que entre los factores de elección ${ }^{20}$ de unos de los modelos de contratos FIDIC, se encuentra la determinación del riesgo y la distribución del mismo en lo relativo al diseño y construcción,

lo que respecta a la imprevisibilidad; creemos que el artículo de mayor relieve es el que pertenece a Dörr Zegers, fundado en que la causa de un contrato bilateral como el de construcción no solo debe existir al instante en que el contrato se forma sino durante todo el proceso de su ejecución en que las obligaciones de cada una de las partes constituye la causa y la razón de ser de las obligaciones de la otra, DÖRR ZEGERS (1985) pp. 253-270.

19 Sobre esto la doctrina alemana del Gemistchte Verträge o contratos mixtos fue la pionera en la materia y en aplicar las teorías de la fusión y absorción contractual de las figuras atípicas.

20 BunNi, Nael G. (1999) pp. 83-86. lo que en definitiva pasa a jugar un papel esencial a la hora de salir a buscar financiamiento bancario para la construcción.

Los modelos FIDIC contenidos en su versión más reciente "1999 Red Book" para obras civiles y de ingeniería, en general, y el específico para proyectos denominados "Llave en mano" el "1999 Silver Book" son solo auxiliares del negocio que se consideran self regulatory agreements ${ }^{22}$ y que pueden tener el carácter de condiciones generales pero exigen que sean adaptados a cada contrato en particular y ajustados al derecho aplicable al lugar donde el contrato se ejecuta ${ }^{23}$.

El uso de estos modelos estándar de contratos de construcción a nivel internacional representa un vehículo seguro para desarrollar inversiones internacionales, en especial en proyectos ejecutados bajo la modalidad EPC y en proyectos contratados bajo la fórmula de diseño, ingeniería y construcción. Diversos países han adoptado estos contratos como el modelo de contratación pública, o se han basado en los conceptos de FIDIC para crear su propio modelo.

El "acuerdo contractual" que proporciona FIDIC en sus formatos (Red Book and Silver Book) viene presidido de definicio-

\footnotetext{
21 Se debe tener presente que existen otros modelos y formatos de condiciones publicados por FIDIC y otros organismos tal como el "1995 Orange Book"; "1999 Yellow Book" y el ICE (Institute of Civil Engeineers) Design and Construct Conditions.

22 Así se conoce la versión FIDIC, BunNi (1999) pp. 503 y ss.

23 Como se sabe una cláusula es condición general cuando está predispuesta e incorporada a una pluralidad de contratos y se dan en la contratación exigiendo que sean conocidas por las partes y que se redacten de forma transparente, con claridad, concreción y sencillez y desde luego, que no sean abusivas.
} 
nes generales que incluyen las condiciones generales y particulares del contrato y su documentación (1.1.), métodos o pautas de interpretación (1.2) y un esquema general (carta aceptación-adjudicación) que estructura desde el acceso oportuno a los terrenos ("The employer" 2.1 y sus licencias y permisos $2.2 \mathrm{y}$ las rutas de acceso 4.15), el ingeniero y su autoridad (3.1); las condiciones naturales o físicas imprevistas (4.12); la relación contractual entre las partes, mandante, ingeniero, contratista, subcontratistas designados (5.1) fecha de comienzo, atrasos; extensiones y aumentos de plazos, suspensiones (todos el apartado 8.1 hasta 8.12 ) certificado de cumplimiento (test on completion 9.1), cuando el mandante se hace cargo (taking over 10.1) defectos y responsabilidades solidarias en caso de operar a través de consorcio ${ }^{24}$ o joint ventures (11.1); derechos a variaciones o cambios de la obra (13.1) y ajustes de precio; pagos, anticipos y retenciones (14.1); riesgos y responsabilidades (17.1) seguros (18.1) y la fuerza mayor que incluye el hecho "imprevisible" (19.1), reclamaciones y arbitraje (20.1), conceptos que facilitan el financiamiento bancario de estos proyectos contribuyendo a su difusión.

En su versión más reciente (1999) se dividen sustancialmente los modelos en cuatro categorías, dependiendo el tipo de obra que acomete, entre los cuales destaca el contrato "llave en mano" (Silver Book) ${ }^{25}$, orientadas al suministro de plantas de todo tipo u obras complejas de cualquier naturaleza, que deben entregarse listas para iniciar

\footnotetext{
24 Werremeyer (2006) p. 251.

25 Huse (2002) p. 56: "For this reason, the Silver Book generally satisfies the lenders requirements that each event resulting in the contractor's right to request an extension of time be specifically identified and defined in the contract".
}

su operación y en las que, por lo tanto, el contratista provee todo lo que sea necesario para que a su entrega el comitente deba operarla" 26 .

\section{LOS CONTRATOS "LLAVE EN MANO" MODALIDAD "EPC"}

\subsection{Concepto del contrato "llave EN MANO" \\ De los distintos métodos de reali-} zación de proyectos que han aparecido en nuestro medio, pero principalmente en el ámbito del comercio internacional -como consecuencia de los avances tecnológicos-, el que mejor refleja las transformaciones experimentadas en este sector es, sin duda el método que por la funcionalidad que busca garantizar se denomina en nuestro medio como contrato modalidad "llave en mano" ${ }^{27}$, conocido y aplicado, especialmente para la construcción de obras inmuebles de infraestructura.

La idea rectora del contrato "llave en mano" nació en el ámbito de los contratos de ingeniería ${ }^{28}$, donde se exigía algo más que un proyecto, dirección y construcción.

26 Podetti (2004) p. 149.

27 Huse (2002) especialmente pp. 17-28; Bartolmew (1998); Ice-Institution of Civil Engineers (2011); Fidic (2005) Bunni, Nael G. (2005); FIDIC (1999c); Collier (1987); Werremeyer (2006); Chern (2010); Jenkins (2006); Cornes (1994); Smith, Currie \& Hancock's (2011) p. 31; Hewitt (2011); Trauner (1990); Rubin, et al (1999); O’Brien (1998); Pinnell (1998); Abeynayke (2010); Scriven (2011); Murdoch (1998); Pakistan EngineERING Council (2010). Cabe hacer notar que muchas de las obras inglesas y americanas no están hechas por abogados, sino por ingenieros como lo hacen constar sus autores.

28 En los Estados Unidos, el proyecto de la autopista 1-15 en la ciudad de Salt Lake, Utah, que se estructuró como un gran contrato EPC, permitió al Departamento de Transporte del Estado construir 
Este "algo más" era ni más ni menos que el perfeccionamiento y entrenamiento para poder hacer funcionar correctamente un emprendimiento complejo, como podría serlo, por ejemplo, una planta de tratamiento de agua, un sistema de comunicaciones o un complejo habitacional con su infraestructura urbana.

Veamos en concreto esta modalidad contractual.

\subsection{Contrato Bajo modalidad epC \\ El contrato "llave en mano" (Design} and Build or Turnkey) toma el nombre de la modalidad EPC, cuando el contratista asume las obligaciones propias del constructor (construction), adquiere los suministros (procurement) y desarrolla la ingeniería del proyecto (engineering) ${ }^{29}$.

\subsection{OTRAS MODALIDADES}

Lo que resulta, sin duda, de interés es la modalidad que convienen algunos contratistas con el mandante con arreglo al principio de autonomía de la voluntad, por

autopistas por un gran valor para preparar la ciudad para los Juegos Olímpicos de Invierno con el sistema tradicional de diseño-licitación-construcción probablemente hubiese llevado mucho más tiempo. El sistema EPC fue la única solución práctica para el Estado en ese caso. Hay otros ejemplos como el Eurotunnel, citados por la mayoría de los autores.

29 En Argentina el hoy derogado Decreto 525 de 15 de marzo de1985, describía este contrato en términos análogos, señalando "ARTÍCULO 3․- A los fines del presente Decreto se entiende por "Llave en mano" la construcción de la obra, la provisión e instalación de los elementos o bienes respectivos, el manejo y supervisión del montaje, la provisión del método operativo, la asistencia en la puesta en marcha y el entrenamiento del personal necesario para su funcionamiento, cuando correspondiere, y todo otro servicio que resulte necesario para la concesión del bien objeto del contrato. Las plantas industriales pueden no incluir su construcción pero sí los demás elementos antes indicados". las ventajas significativas para este último, como son los contratos de rendimiento garantizado de producción y el más sofisticado de todos, el denominado "de producto en mano" 30 .

Bajo esta modalidad desaparece la tradicional relación tripartita que se da en el contrato de obra a suma alzada, en general (design bid build projects), entre cliente, ingeniero y contratista, para quedar sustituida por una única relación entre Mandante o Comitente y Empresario o Contratista (One Stop Shopping One point of Contact), elegido por licitación u otra forma de selección ${ }^{31}$

30 Podetti (2004) pp. 249-250. Cabe destacar que la primera modalidad persigue asegurar determinados rendimientos, en tanto que la segunda se asemeja a una compraventa de cosa futura "conforme a los requerimientos del producto solicitado".

31 Una variante del contrato EPC, aunque este método de contratación es más corriente en el sector minero, es el uso de los contratos EPCM que se ha vuelto cada vez más frecuente en grandes proyectos de infraestructura y de construcción. Al parecer el significado de EPCM (en comparación con EPC) sigue siendo relativamente desconocido en el ámbito de la construcción. La confusión fundamental estriba en que si bien la "C" en "EPCM" significa "construcción", se refiere al contexto de "CM", es decir, a la Gestión de la Construcción. ICEInstitution of Civil Engineers (2011) p. 100: "EPC (Engineering, Procurement and Construction): means the company is contracted to provide engineering, procurement and construction services by the owner. Design \& Construct style contracts, where the project is largely Contractor managed and the cost risk and control are weighted towards the Contractor and away from the Owner. The EPC contractor has direct contracts with the construction contractors. EPCM (Engineering, Procurement and Construction Management): means the company is contracted to provide engineering, procurement and construction management services. Other companies are contracted by the Owner directly to provide construction services and they are usually managed by the EPCM contractor on the Owner's behalf. Professional Services contracts, where the project is largely Owner managed and the cost risk and control is weighted towards the Owner". 


\section{4. ¿TíPICOS O ATÍPICOS?}

En el Derecho Comercial moderno, existe la tendencia a dar cabida a contratos denominados "atípicos" elaborados al amparo de la autonomía contractual y al compás del proceso de especialización y que se desplazan desde el mundo anglosajón, manteniendo incluso su denominación de origen, como sucede con el leasing, el factoring, el franchising, los contratos de colaboración empresarial en materia de construcción o aquellos que recaen en transferencia de tecnología, secretos industriales o de financiación o riesgo compartido como ocurre con el joint-venture, el know-how, el engeneering, etc.

$\mathrm{La}$ internacionalización que alcanza la economía y el fenómeno de la globalización apoyado en medios tecnológicos nuevos, contribuyen a incrementar la cantidad de bienes y servicios que produce una economía y con ello la utilización masiva de estos contratos que muchas veces escapan a los moldes tradicionales.

En términos generales, el contrato "llave en mano" o "turnkey contract" modalidad EPC es un contrato atípico en que el contratista se obliga frente al cliente o contratante, a cambio de un precio, generalmente fijo o alzado, para construir y poner en funcionamiento una obra determinada que él mismo previamente ha proyectado pero que escapa en su conjunto a las categorías clásicas del contrato de construcción sin perder su sustancia.

Es en este orden de ideas en que el principio de la autonomía de la voluntad tiene plena eficacia con el objeto que los contratantes puedan crear contratos que regulen sus intereses particulares.

Como es obvio, estos contratos deben considerar los elementos esenciales y comunes a todo contrato, como son los ele- mentos de la esencia, tales como, capacidad, validez, voluntad no viciada, objeto y causa lícita. No debemos olvidar que el contrato atípico es un contrato y que obliga en dichos términos a las partes contratantes.

Algunas veces, por inducción, estos contratos participan del régimen jurídico de los contratos en general; otras veces, apenas se acomodan.

En un buen número de casos, estos contratos se presentan como actos jurídicos "enganchados" o "combinados"; cuyas modalidades de ejecución dan lugar a una verdadera operación cesárea, difícil de encasillar en un régimen jurídico unitario. En buena medida su caracterización se resuelve a partir de su funcionamiento práctico, describiendo la operación, tal y como ella es practicada en nuestro medio, para luego confrontarla con el ordenamiento jurídico y sacar las conclusiones correspondientes en cuanto a su validez, naturaleza jurídica y demás aspectos legales.

Como dice un fallo arbitral en esta hipótesis, "la noción de tipificación de los contratos o, lo que es lo mismo, la existencia de una categoría de contratos calificados tradicionalmente como "nominados", alude a contratos en los cuales las normas jurídicas han creado unos estatutos estándar, que permiten a los contratantes ahorrarse el esfuerzo de convenir todos y cada uno de los efectos que desean obtener, por medio del convenio que han celebrado. De este modo, si las partes han celebrado un contrato de compraventa, no necesitan expresar cuáles son las obligaciones del comprador y del vendedor, ni las responsabilidades que este último adquiere respecto de la calidad de lo vendido, o en la defensa del dominio que dice transferir al comprador. Bastará recurrir al Título XXIII del Libro IV del Código Civil para conocer los derechos, obligacio- 
nes y cargas que resultan para cada uno de los contratantes" 32 .

En resumen el contrato "llave en mano" se presenta como un contrato enganchado al contrato general de construcción combinando elementos distintos cuyas modalidades de ejecución dan lugar a un régimen complejo de encasillar, pero que mantiene los elementos que conforman esta tipología.

En esencia sigue siendo, entonces, un contrato bilateral, oneroso, conmutativo y de ejecución diferida en el tiempo, a suma alzada ajustable en tanto que de buena fe el mandante y el constructor asumen obligaciones recíprocas, gravándose cada uno en beneficio del otro.

\subsection{CONTRATO INNOMINADO}

En nuestro medio, aunque ampliamente conocidos en el mercado, los contratos llave en mano modalidad EPC pertenecen a la categoría de los denominados contratos "innominados" 33 , toda vez que no se encuentran regulados expresa y sistemáticamente en el Código Civil como tampoco en el Código de Comercio ni en ningún otro texto legal de nuestro ordenamiento jurídico.

Con ello cobra plena eficacia el principio de la libertad contractual ${ }^{34}$, recogido

32 Andrés Cúneo M., Sentencia 29 de julio de 2010 , CAM Santiago Rol 1087-2009. Considerando 36.

33 Andrés Cúneo M., Sentencia 29 de julio de 2010, CAM Santiago Rol 1087-2009. Considerando $42^{\circ}$ que, en cambio, por su extensiva aplicación en la práctica, lo encasilla en la clasificación de los contratos nominados.

34 Esto mismo permite la utilización de esta modalidad bajo distintos supuestos contratos "llave en mano" mixtos: cuando una parte del proyecto es tradicional y la otra es llave en mano; contratos "llave en mano" parciales: cuando se contrata de forma tradicional todo el proyecto pero de subcontrata una por los arts. 12 y 1545, del Código Civil, sin perjuicio de lo cual se le cobija al amparo del contrato de construcción y se aplican supletoriamente las reglas generales que se imponen a todos los contratos cuyos elementos esenciales y de la naturaleza si bien pueden no encontrarse en este caso en particular en la ley, sí aparecen en la costumbre, la cual se aplica, en este caso, en virtud del art. 1546 del Código Civil que remite a la costumbre como elemento integrante de las obligaciones y, también, según dispone el inciso tercero del art. 1563 del mismo Código. Al considerar de este modo las cláusulas de uso común, que se presumen integrar el contrato, sin necesidad de que se expresen.

\section{CARACTERÍSTICAS PRINCIPALES DE LOS CONTRATOS “LLAVE EN MANO"}

Del concepto anterior se desprende que los dos rasgos esenciales de los contratos "llave en mano" son los siguientes:

\subsection{Fusión DE LAS ETAPAS DE ELABORACIÓN DEL PROYECTO Y EJECUCIÓN DE LA OBRA EN UNA SOLA PERSONA \\ Se llama precisamente "llave en} mano", ya que el contratista desarrolla, dirige y emprende el proyecto $^{35} \mathrm{y}$, al finalizarlo,

parte llave en mano; contratos semi "llave en mano": cuando el contratante celebra varios contratos siendo uno de ellos llave en mano y encarga a este último contratista la coordinación de los demás contratos.

35 Smith, Currie \& Hancock's (2011) p.111. Existe sí una importante salvedad que es la suficiencia y adecuación del diseño, ya que en los EstadosUnidos de N.A, se aplica en caso de deficiencia en los planos la Spearin Doctrine "The contractor is bound to build according to plans and specifications prepared by the 
lo entrega y el mandante, por su parte, recibe "la llave" de la obra, a la espera de la entera satisfacción del mandante o comitente que la encarga.

Cabe destacar que en este tipo de contrato la intervención del mandante es mínima ya que el ingeniero y las instrucciones que transmite por cuenta de quien representa ${ }^{36}$, se limitan a "funciones de vigilancia respecto de la adecuada ejecución de la obra" (Hands off" approach to Project) ${ }^{37}$.

"Estas facultades del ingeniero (en caso del contrato "llave en mano EPC" deben limitarse clara y exclusivamente a funciones de vigilancia respecto de la adecuada ejecución de la obra. De lo contrario se desnaturaliza una de las características esenciales del contrato de construcción, la de inexistencia de subordinación jurídica y técnica" ${ }^{38}$.

En consecuencia, la estructura sobre la que descansa el contrato "llave en mano" modalidad EPC, y que ha revolucionado ciertamente la industria de la construcción, implica para el Mandante una pérdida de control sobre el proyecto y una reducción o dispensa considerable en las funciones del ingeniero $^{39}$. En este tipo de contratos actúa

owner, the contractor wil not be responsible for the consequences of defects in the plans and specifications".

36 FIDIC (1999c) puntos 3.1 a 3.5

37 "This EPC/Turnkey Bidding Document may be suitable for the provision on turnkey basis of a process or power plant, of a factory or similar facility, or of an infrastructure project or other type of development. In these Bidding Documents the word "Engineer" has been replaced with the "Project Manager/Engineer" to maintain the approach of EPC/Turnkey type of Contracts".

38 Podetti (2004) p. 157. Cabe señalar que en la propia FIDIC CONTRACTS GUIDE (1999) p.12, se indica que: "If the employer intends to supervise closely or control the contractor's work, or to review most of the construction drawings. With the greater extent of Contractor's risks, he needs to have greater freedom of action and less interference by the Employer". 39 Huse (2002) p 125. generalmente solo un representante del mandante (employer's representative), siendo posible incluso en los casos más extremos que se prescinda completamente de su participación, como veremos ${ }^{40}$.

El contratista asume como contrapartida y en consecuencia, mayores riegos que en la forma habitual del contrato de construcción, pero sin llegar a responder, como es lógico, por los casos de fuerza mayor.

En resumen, los contratos de construcción bajo el régimen "llave en mano" incorporan la ingeniería, el suministro y la producción (EPC) que, como veremos, en el contrato implica que la obra no solo será diseñada, construida, sino también equipada y puesta en marcha por el contratista sin intervención del mandante ("Hands off"). En tal condición, el contratista asume dicho compromiso sin que obste por cierto a que, en la práctica, pueda dividir internamente esta labor con otros subcontratistas ${ }^{41}$.

\subsection{Obligación Que asume el CONTRATISTA}

En este tipo de contrato el énfasis ha de ponerse en la responsabilidad global que asume el contratista frente al cliente o mandante. En efecto, frente al mandante el contratista debe entregar una obra completamente equipada y en estado de funcionamiento.

Como hemos señalado, los contratos "llave en mano" incluyen la ingeniería, el suministro y la producción (EPC) que implica que la obra no solo será diseñada,

\footnotetext{
40 En eso los modelos FIDIC (Orange, Red, Yellow Books) operan de una forma más o menos similar en cuanto a las funciones excepto en los modelos del Silver Book en que el ingeniero juega un rol mucho más reducido.

41 One company can do the engineering; another can do the procurement, while still another can do the construction project management.
} 
construida, sino también equipada y puesta en marcha por el contratista que asumió dicho compromiso. Lo anterior, en la mayoría de los casos puede tener como efecto que las ofertas de adjudicación (bid tender) tiendan a ser sumamente complejas por lo que empuja hacia formas más directas de elección del contratista.

La modalidad EPC implica que el mandante se debe concentrar exclusivamente en el financiamiento de las obras, asumiendo como obligación esencial la de entregar, en forma oportuna, el pleno y oportuno acceso a los terrenos o las servidumbres (sin retardo) y toda la información disponible concerniente al expedito acceso a los mismos y del lugar donde se emplazará la obra.

Refiriéndose a este elemento la doctrina alemana ha sostenido que se entiende por "esencia" (das Wesen) ${ }^{42}$ de un contrato, aquellos que se refieren a "la estructura interna fundamental" que presenta este tipo normativo y se concreta en preservar aquello que pudiera estar en contradicción con los principios que configuran y que integran el tipo. Son por lo tanto, los principios informadores o "directores" (Karl Larenz) de la concreta regulación jurídica y a los que debe atenerse el intérprete.

La concentración como una sola tarea del diseño, el suministro y la construcción permite un desarrollo más veloz ${ }^{43}$ $y$ mayor certeza en cuanto al costo general y una ventaja en cuanto a que se entrega "lista para su utilización, operación y funcionamiento". Ello representa una gran ventaja para obtener el financiamiento bancario de proyectos de infraestructura.

\footnotetext{
42 Duque Domínguez (1991) pp. 1 a 109.

43 Como ventajas de estos contratos bajo el método turnkey se dice que se encuentra el fast track que permiten proceder aun cuando el diseño no esté completo.
}

La gran desventaja, en cambio, se presenta en la pérdida de control y supervisión del ingeniero a cargo que pasa a ser reemplazado, como hemos dicho, por un empleado o agente del mandante.

\subsection{Existencia de un solo CONTRATO Y MODALIDADES DEL MISMO \\ A diferencia del contrato de cons-} trucción de obra general la celebración de un contrato "llave en mano" importa la celebración de un solo y único contrato realizado entre el mandante y el contratista. Generalmente, en la selección de este tipo de contratos ejerce una influencia decisiva la tecnología implicada en el proyecto que se pretende realizar y que se va a manifestar no solo en los planos y especificaciones técnicas sino también en los derechos de propiedad industrial implicados en el proceso de producción y, en determinados casos, en la formación de personal y en la asistencia técnica proporcionada por el contratista.

Ahora bien, el contrato se puede celebrar bajo las modalidades "llave en mano"; "producto en mano" y "mercado en mano". Cuando se conviene bajo la forma de llave en mano, es un contratista único el que cumple las obligaciones relativas al diseño de la obra o de sus partes (por ejemplo, la construcción de las turbinas) a la transferencia de tecnología, al suministro del equipo y los materiales, a la instalación del equipo y a la construcción de las obras de ingeniería civil, las que regularmente se entregan con plena capacidad de funcionamiento. Esta última figura toma en el ámbito comparado el nombre de "Contrato de Engeneering" ${ }^{44}$.

\footnotetext{
44 Fournier (1981) p.1 Consiste en que una empresa especializada en la materia traspasa, a cambio de una retribución, a favor de un adquirente, ciertos
} 


\subsection{Plazos de término y eXtensiones} de tiempo de EjeCuCión. REFERENCIA A LA DOCTRINA “TIME AT LARGE"

En materia de contrato general de construcción, el cumplimiento de los plazos de ejecución de la obra resulta un factor determinante para obtener el resultado esperado, sin perjuicio de la incidencia que presenta en el contrato ajustado por precio alzado, en el que normalmente, el mandante paga al contratista, según estado de avance, esto es por etapas que se distribuyen en el tiempo.

En principio, en el contrato de construcción los plazos contractuales se establecen en beneficio reciproco ${ }^{45}$, atendido a que ambas partes van programando el cumplimiento de sus obligaciones, tanto en la etapa de ejecución como en la recepción de la obra.

Se trata, por lo consiguiente, de no provocar alteraciones que puedan causar daños o perjuicios por atraso, existiendo, no obstante, causas justificadas o estipulaciones contractuales que autorizan el aumento o la suspensión de los plazos en curso y que dilatan la conclusión del contrato, con las consecuencias económicas que siguen, como es lógico, a la mayor extensión de estos plazos.

También resulta de interés destacar que en el contrato de construcción el otorgamiento de plazos puede ser bajo la modalidad de un plazo expreso o plazo tácito de forma que previo a su expiración no existe retraso en el cumplimiento de la obligación.

conocimientos técnicos o elabora un proyecto de estudio o proporciona algunos equipos y, a veces, se encarga de la ejecución misma de una obra o de la instalación industrial de los equipos y maquinarias suministradas, garantizando su adecuado funcionamiento.

45 Podetti (2004) p. 232.
Este último aparece asociado a la naturaleza o del objeto que se espera con la obra encomendada, lo cual supone un plazo explícito o tácito indispensable para cumplir dicha obligación (art. 1494 del CC) o "razonablemente necesario para concluirla" 46 .

Interesa en esta parte detenerse en el concepto extraído del ámbito de la jurisdicción del common law anglosajón que acepta invocar la extensión del tiempo para completar el contrato con ocasión de un acto de prevención de parte del mandante (prevention principle) y que puede tener como consecuencia la aplicación de la doctrina denominada "time at large" 47 .

Ella describe la situación cuando el contrato convenido -especialmente cuando no se contempla un mecanismo expreso de extensión de plazo- no puede ser concluido en la fecha específica acordada por razones o eventos que no son imputables al contratista, como por ejemplo, sucede con la falta de acceso al terreno o problemas con la entrega del proyecto técnico, de forma tal que el plazo para ejecutarla se vuelve ineficaz o imposible de determinar mediante una extensión y el mandante o comitente pierde el derecho a cobrar perjuicios por el retardo -obviamente hasta ese momento-, toda vez que no existe una época cierta desde que se puedan cobrar.

\footnotetext{
46 Código Civil argentino, art.1635.- A falta de ajuste sobre el tiempo en que debe ser concluida la obra, entiéndase que el empresario debe concluirla en el tiempo razonablemente necesario, según la calidad de la obra, pudiendo en tal caso el locatario exigir que este tiempo se designe por el juez.

47 Véase Newsletter 21-8-2009 de SMith (2009) "The prevention principle, time at large and extension of time clauses". Disponible en: http:// www.lexology.com/library/detail. Con abundante cita de casos judiciales de revisión "open up, review and revise”. Véase Bellhouse y CoWAn (2008); AtKinson, Daniel (2007)
} 
En tal caso, por vía judicial se debe establecer un plazo razonable para su terminación (reasonable time for completion).

Si bien la aplicación de esta doctrina no se ajusta al sistema continental de obligaciones ${ }^{48}$, sin embargo los principios y reglas en que se asienta sí tienen presencia, especialmente cuando se trata de retardos provenientes de causas externas, principalmente del mandante o dueño de la obra ("fait du maitre de l'ouvrage") tales como la buena fe, la equidad, el enriquecimiento sin causa $^{49}$, y los principios generales de

${ }^{48}$ El Código Civil del Perú permite la reducción judicial de la cláusula penal en su artículo 1346 al disponer que "El juez, a solicitud del deudor, puede reducir equitativamente la pena cuando sea manifiestamente excesiva o cuando la obligación principal hubiese sido en parte o irregularmente cumplida". Algo parecido sucede en Francia en que el art. 1147 del Code Civil que dispone "Le débiteurest condamné, sil y a lieu, aupaiement de dommages et intérêts soit à raison de l'inexécution de l'obligation, soit à raison du retard dans l'exécution, toutes les foisquiline justifie pas que l'inexécution provient d'une cause étrangère qui ne peut lui être imputée, encoré quill n'yait aucune mauvais foi de sa part".

49 Este punto aparece recogido en la Sentencia de la I. Corte de Apelaciones de La Serena, de fecha 13 de enero de 2012, autos Rol Rol 1670-2011.- (Civil). Casación y Apelación: "QUINTO: Al respecto la demandada tanto en la presentación del recurso de apelación cuanto como alegación o defensa efectuada en esta instancia y, asimismo en estrados, advirtió que no concurrían en el caso sub lite todos los requisitos o presupuestos que conforme la doctrina hacen procedente la acción de repetición o in rem verso. Señaló al efecto el articulista de la demandada que se requería para su admisión, I el que una persona experimente un empobrecimiento, II el que otra persona obtenga un enriquecimiento, III una relación entre ambos hechos, IV carencia de causa y, $\mathrm{V}$ que no exista otra acción o que ella sea subsidiaria. Otros tratadistas señalan que los requisitos para su procedencia solo serían la adquisición de una ventaja patrimonial para el demandado, y correlativo empobrecimiento del actor, conexión entre enriquecimiento y empobrecimiento y falta de causa que justifique el enriquecimiento. Respecto de los derecho en los que se encuentran elementos comunes que participan de esta relevante doctrina argumental.

cinco requisitos señalados por el demandado señala que en lo pedido por el actor, no concurrirían los dos últimos presupuestos. Esto es carecer de causa, entendida en el sentido de antecedente jurídico que justifique el beneficio obtenido y el perjuicio sufrido. Y que en este caso existe entre las partes una relación patrimonial que impediría prosperar a la acción in rem verso. Refiriendo que este requisito, el actor ha expuesto en estrados que en la especie ha habido un contrato, pero que el empobrecimiento no se deriva de aquel, sino de obras mayores y extraordinarias asumidas, de buena fe por su parte. Lo cual hace a su juicio que se entienda cumplido el cuarto requisito. Con relación al último de ellos, que la acción in rem verso sea subsidiaria significa a juicio del demandado que, no es posible recurrir a ella sino a falta de toda otra acción que permita obtener reparación. De manera tal que si la ley ha otorgado al empobrecido otra acción, debe este sujetarse a ella y, no a la de repetición. Y que en el caso de autos, se da la situación que, quienes han dado origen a las obras mayores son justamente, terceras personas que son aquellas que extraen material granular del lecho del río, y contra ellas debería dirigirse el actor. Respondiendo el actor, en orden a que los areneros no se han enriquecido con aquello, y que el único beneficiado en la especie es el Serviu IV Región de Coquimbo, siendo procedente la actio in rem verso incoada, que es una situación de excepción cuando concurren sobre un mismo supuesto normas reguladoras de responsabilidad civil y enriquecimiento, puede acudir a la que estime más adecuada $\mathrm{y}$, además atento a la circunstancia que el derecho no tolera la inequidad; SEXTO: Que consecuencia de lo ya analizado es que solo resta verificar que, en el caso sub iudice, los elementos dubitados del enriquecimiento injustificado, se encuentren presentes en la circunstancia que origina esta pretensión. Con respecto a la ausencia de causa sea del caso establecer que todo hecho tiene una causa, y de lo que se trata es de exigir a todo enriquecimiento o más ampliamente a toda atribución patrimonial, una causa jurídicamente justificante que en derecho sea aceptable. Como lo señala el profesor de Derecho Civil de la Universidad de Concepción Daniel Peñailillo Arévalo, en su publicación citada "la circulación de bienes y ventajas y para el ingreso de un provecho en un patrimonio, 


\subsection{Presupuestos necesarios Para QUe} EL CONTRATO SE PONGA EN MARCHA

Debe quedar claro que si bien bajo la modalidad EPC el contratista dispone de amplia libertad para desarrollar la obra con plazos previstos, ello sucede estrictamente en la medida en que se cumpla con todos los presupuestos de la modalidad contractual que se elija, esto es, que se asegure el acceso oportuno a los terrenos donde se va a emplazar ${ }^{50}$; que la información sea fidedigna y que el objeto de la misma no experimente modificaciones ni aumentos de obras en su desarrollo y ejecución que requieran de una mayor extensión.

En otras palabras, y siguiendo el modelo propuesto por FIDIC el computo inicial de los plazos de ejecución de estos contratos y las suspensiones de los mismos (FIDIC 8.1 a 8.12) están subordinadas, en primer lugar al acceso que da el mandante o comitente (The Employer) en tiempo y forma a los terrenos (Right of Access to the

el Derecho prevé y reconoce dos caminos; el negocio jurídico como vehículo de la libertad y autonomía privada en la autorregulación de sus intereses, y la ley, que concreta principios de justicia, prudencia y seguridad. Cuando la adquisición de un bien o una ventaja no sigue los cauces previstos en el ordenamiento jurídico (el negocio o la ley) se dice que esta adquisición no tiene causa. (José Álvarez Caperochipi. El Enriquecimiento sin causa, Santiago de Compostela 1979, pág. 44). Y en este caso, es inconcuso que el enriquecimiento del Serviu IV Región de Coquimbo, que cuenta con una obra mayor, no se debe a un acto jurídico sino a hechos diferentes a la voluntad de la demandante".

50 Pakistan Engineering Council (2010): "17.1 Access to and Possession of the Site: The Employer shall in reasonable time grant the Contractor access to and possession of the Site, which may, however, not be exclusive to the Contractor. The Employer shall to the extent stated in the Specification provide means of access for delivery of all Plant and Contractor's Equipment to the Site".
Site, FIDIC 2.1 $)^{51}$ contando con todas las aprobaciones, documentos de respaldo y permisos correspondientes (FIDIC 2.2) y recién entonces se puede contar el plazo de ejecución de la obra ${ }^{52}$.

En caso contrario, el efecto inmediato es que los plazos se extienden y aumentan, adicionalmente por variaciones o aumento de obra ordenados por el mandante (Extention of Time for Completion, FIDIC 8.4).

Por su parte, el contratista se encuentra facultado y puede solicitar una extensión de plazo por una serie de causas que lo justifican entre las que se encuentra el incumplimiento del mandante, la fuerza mayor o los trabajos adicionales requeridos por este a través de órdenes de variación del proyecto (Variation Orders), entre otras ${ }^{53}$.

51 Huse, Joseph A. (2002) p. 106: "The employer duty to give access to and provide the site constitutes one of the fundamental aspects of the parties relationship under the construction contract. Whether or not it is specifically included as an obligation in the construction contact, the employer will nonetheless have to provide the contractor access to and possession of the site... Contractor should be adequately protected from the consequences of the employer's failures to provide site access and possession and to provide certain elements of the site at the appropriate time or times during the construction process". Igual cosa sucede (6-03 p 107) con los permisos y licencias "Employer assistance with permits and licenses".

52 FIDIC (1999c), p. 8: "Site" means the place or places, provided or made available by the Employer where work is to be done by the Contractor or to which Plant is to be delivered, together with so much of the area surrounding the same as the Contractor shall with the consent of the Employer use in connection with the Works otherwise than merely for the purposes of access"., Obligations of the Employer.

53 Huse (2002) p. 58: Con relación al Silver Book Sub Clause 20.1: \#Despite the general rule that the contractor is responsible for achieving completion on a certain date, it is commonly accepted that the contractor will not be responsible for delays caused by certain types of events outside his control". 


\subsection{PROCEDIMIENTO DE LiCiTACIÓN DEL} CONTRATO

El hecho de que en los contratos "llave en mano", el contratista asuma la concepción y la ejecución de la obra condiciona no solo el procedimiento de adjudicación del contrato, generalmente un procedimiento restringido o negociado, sino también la determinación del objeto y la función del cliente o de su ingeniero.

Hay que tomar en consideración que el establecimiento de un precio a suma alzada obliga al mandante a no poder variar prácticamente nada una vez realizada la adjudicación salvo acuerdo, con el constructor o contratista.

\subsection{Elaboración del proyecto y DISEÑO DE CONSTRUCCIÓN}

Como se podrá advertir, a diferencia de los contratos tradicionales de construcción, en los contratos "llave en mano" la elaboración y descripción detallada del proyecto tiene lugar una vez que el contrato se va a ejecutar, circunstancia que justifica conceder al contratista un derecho a introducir modificaciones en sus planos, a su propio coste y riesgo y siempre que se respeten los parámetros contractuales acordados (calidad, cantidades de materias primas, rendimientos). Sin embargo, para que esta modalidad sea plenamente eficaz, es indispensable que el mandante limite el derecho a formular modificaciones o variaciones a la obra en algún momento ya que en el evento que no cumpla con esta obligación, no podrá exigir, al contratista, el cumplimento del contrato dentro del plazo pactado ${ }^{54}$.

\footnotetext{
54 The Contractor shall submit to the Engineer for approval: (a) within the time given in the Contract or in the Program such drawings, samples, models or information as may be called for therein, and in the
}

\subsection{Existencia de un precio} DETERMINADO A SUMA ALZADA

La obligación global que se deriva de los contratos "llave en mano" para el contratista influye de manera decisiva en la determinación del precio, que no puede ser, más que un precio alzado. Esto se justifica por la necesidad de buscar entidades que financien la obra, que puede estar sujeta a contingencias y variaciones en los precios estipulados que por su magnitud pueden desembocar en "conflictos de difícil solución que ocasionan mayores demoras y costos que si hubieran establecido sistemas contractuales más equitativos, como los reflejados en los modelos FIDIC"55.

De ahí que sea frecuente en este aspecto que se ajuste el precio tanto en Chile como en el extranjero bajo la modalidad de la suma alzada ${ }^{56}$ (Lump sum Price basis) $)^{57}$ y que es pagadera por estado de avances.

numbers therein required, and (b) during the progress of the Works, such drawings of the general arrangement and details of the Works as specified in the Contract or as the Engineer may require. The Engineer shall signify his approval or disapproval thereof. If he fails to do so within the time given in the Contract or the Program or if no time limit is specified, within twenty eight (28) days of receipt, they shall be deemed to be approved. Approved drawings, samples and models shall be signed or otherwise identified by the Engineer. The Contractor shall supply additional copies of approved drawings in the form and numbers stated in the Contract.

55 PodetTi (2004) p. 151.

56 Así por ejemplo se recoge en el Código Civil argentino el cual en su art. 1633 señala: "Aunque encarezca el valor de los materiales y de la obra de mano, el locador bajo ningún pretexto puede pedir aumento en el precio, cuando la obra ha sido contratado por una suma determinada".

57 FIDIC: "4.1 Except as otherwise expressly provided under the Conditions of Contract, the unit rates and lump sum amounts entered in the Schedule of Prices will be the rates at which the Contractor will be paid, and shall be deemed to include for the full scope and all costs incurred by the Contractor in the performance of the Works, the provision of services including his 
Para que ello sea efectivo, se requiere que se cumplan las condiciones de precio global fijado de antemano e invariable para la totalidad de los trabajos previstos en los planos y presupuestos.

El contrato "llave en mano" ajustado a suma alzada significa en definitiva, que el precio de la obra se fija, como hemos dicho, a un tanto global y requiere, por lo tanto una invariabilidad en el curso de la obra que corresponda a esa invariabilidad en el precio.

La posibilidad de ajustar el precio del contrato se produce, en cambio, precisamente cuando el contratista se ve obligado a incurrir en mayores costos asociados a la construcción de la obra por causas que no le son imputables. De esta manera, tratándose de un contrato conmutativo, con el objeto de mantener la equivalencia de las prestaciones recíprocas y evitar que una parte se enriquezca a costa de la otra se debe, en tal caso, ajustar el precio y extender los plazos para su ejecución.

\subsection{El estándar de conducta} EXIGIBLE AL CONTRATISTA BAJO LA MODALIDAD DE CONTRATO "LLAVE EN MANO O TURNKEY-EPC"

Como anota el reconocido y tantas veces citado autor inglés Joseph Huse ${ }^{58}$, en contraste con los proyectos tradicionales de

overheads, income tax, super tax, other indirect costs, customs \& other duties, profits and costs of accepting the general risks, liabilities and obligations set forth or implied in the Contract, except for such costs which are specified as reimbursable under the Contract". Por su parte, Huse, Joseph A. (2002) 4-38, p. 59 referido al Silver Book sub clause 1.1.4.1: " the agreed amount stated in the contract agreement for the design, execution and completion of the works and the reminding of any defects, and includes adjustments".

58 Huse (2002) p. 15. En igual sentido véase, Cornes (1994) p. 58. construcción en que las tareas se ejecutan por separado a través de una "interfaz" de entidades por intermedio de "paquetes" que interactúan con diferentes problemas de coordinación y responsabilidades, los Turnkey o EPC hacen al contratista frente al mandante, enteramente responsable decreciendo las posibilidades de reclamo ${ }^{59}$.

Hay que tener en cuenta que el contrato "llave en mano" implica una especialización del contratista así como la obligación de este de entregar un producto terminado. Por esta razón se asume una obligación global de realizar todas las prestaciones y emplear todas las "reglas del arte" necesarias, coadyuvantes o complementarias de la obra a realizar.

Pero para que ello funcione, como modalidad "llave en mano" EPC se supone en primer lugar que existan especificaciones o estándares de desempeño de rendimiento y de diseño preestablecidos, ya que el mandante, como hemos dicho, no interviene o no debe intervenir en forma directa para asegurar una mejor coordinación sistémica.

Siguiendo con el autor inglés Huse ${ }^{60}$, podemos sostener que la circunstancia que la responsabilidad aparezca unida en la persona del Contratista (single point responsibilty) supone dos consecuencias, a saber: el criterio de ejecución (perfomance criteria) y el estándar de conducta esperable del contratista (standard perfomance).

\section{"The contractor must design and build the Works in a manner such that the performance of the finished Works satisfies the criteria set out in the con-}

\footnotetext{
59 Huse (2002): “When he looks for accountability as to performance and quality of the works, he need to look no further than the contractor". Agrega "without the need to address whether it is a design or workmanship problem".

${ }^{60}$ Huse (2002) pp. 18 y ss.
} 
tract (otherwise known as performance criteria). The employer will define these performance criteria in such a way as to ensure that the Works provide a least level of performance required for profitable production. The performance criteria may specify output, input, waste and any other performances the employer may desire."

Ello supone primero cumplir con las obligaciones de la esencia de este modelo tal como el acceso inmediato y expedito a los terrenos o la constitución de las servidumbres necesarias.

A ello se agrega que la información entregada por el mandante sea fidedigna y que no haya modificaciones o variaciones sustanciales como aumentos o trabajos adicionales de obra que desnaturalicen el modelo EPC, al impedir ejecutar la obra dentro del plazo considerado y previsto como razonable.

Es decir el estándar razonable de un contrato llave en mano EPC se mantiene en la medida en que la información entregada por el Mandante sea la correcta y que se cumpla con los presupuestos fundamentales de este tipo de contrato ${ }^{61}$.

Tomando como base ese estándar -que no asegura ni garantiza un resultado sino un método-, estamos delante de un modelo de conducta profesional de cuidado y diligencia (professional duty of care) que deberá ajustarse a los estándares y prácticas de la industria, ya que cambian en cada contrato en ausencia de una estipulación específica. Tomando en consideración que

61 Huse (2002) 5-64 p. 92, hace notar que en materia de reglas de interpretación de cláusulas de estos contratos, las modalidades establecidas como principios en el Red Book son similares al del Silver Book. se deja en libertad al contratista para dirigir y completar los diseños, el mandante debe aprobar y revisar dentro de plazo el diseño final, reservándose, en esta etapa, como es lógico el derecho a efectuar los cambios que estime convenientes ${ }^{62}$.

Cabe señalar que el modelo de conducta aplicable en tal caso al resultado que se espera y que proviene del Derecho Inglés "fitness for the purpose" o bien "fit for the purpose for which it was made" se recoge expresamente en el Silver Book 1999 de FIDIC (cláusula 4.1) como un estándar de funcionamiento para la industria de este tipo de contratos y que, como hemos señalado, describe el cuidado que debe tener el contratista y el alcance de su responsabilidad en la medida en que estemos delante un contrato "llave en mano EPC".

\section{BIBLIOGRAFÍA}

Abeliuk, René: Las Obligaciones, Santiago, Ediar, 1983

Abeynayke, Mahesh (2010): Construction Law and Dispute Resolution Practice (Verlag, USA)

Atkinson, Daniel (2007): Time At Large, disponible en: http://www.atkinson-law. com/library

Bartolmew, Stuart H. (1998): Construction Contracting Business and Legal Principles (Prentice Hall, USA)

Bellhouse, John y Cowan, Paul (2008): Common Law "Time at Large" Arguments in a Civil Law Context, White \& Case LLP, London; disponible en: http:// www.4newsquare.com/content/Publications/197.pdf

\footnotetext{
62 FIDIC (1999): Asociación Española de Consultores en Ingeniería y Organización, Condiciones de Contratación para Proyectos EPC/Llave en mano (Madrid).
} 
Bunni, Nael G. (1999): The FIDIC Forms of Contract, Chapter 6, pp. 83-86. Disponible en: http//www.FIDIC.org/resources/contracts/courses.asp

Bunni, Nael G. (2005): The FIDIC Forms of Contract: the fourth edition of the Red Book 1992 (Londres, Blackwell).

Chern, Cyril (2010): The Law of Construction Disputes (London).

Collier, Keith (1987): Construction Contracts (Prentice Hall, USA).

Cornes, David L. (1994): Design Liability in the Construction Industry (Blackwell UK).

De Amunátegui Rodríguez, Cristina (2003): La claúsula rebus sicstantibus (Valencia)

Del Arco Torres, Miguel y Pons GonzÁLEZ, Manuel (1991): Derecho de la Construcción (Madrid).

De la Maza, Lorenzo (1933), La teoría de la imprevisión: (en relación con el derecho civil chileno), (Santiago, Universidad de Chile)

Dörr Zegers (1985): "Notas acerca de la teoría de la imprevisión", Revista Chilena de Derecho, vol. XII.

Duque Domínguez (1991): "Escritura, estatutos y límites a la libertad estatutaria en la fundación de las sociedades anónimas" en VV. AA., Derecho de Sociedades Anónimas I, La Fundación (Madrid) pp. 1-109.

FaIRWEATHER, Virginia (1999): Construction Claims: Prevention and resolution (John Wiley, USA, 3rd edit.)

FIDIC (1995) Guide to the Use of FIDIC Conditions of Contract for Design-Build and Turnkey-Design-Build and Turnkey (1995 Orange Book) Guide, (Ginebra, FIDIC).

FIDIC (1999a), Conditions of Contract for Plant \& Design-Build-Yellow book, (Ginebra, FIDIC).
FIdic (1999b): Asociación Española de Consultores en Ingeniería y Organización, Condiciones de Contratación para Proyectos EPC/Llave en mano (Madrid, FIDIC).

FIDIC (1999c): Conditions of Contract for Construction (Ginebra, FIDIC).

FIDIC (2005), The Fidic Contracts Guide (Construction, Plant and Design-Build and EPC/ Turnkey Contracts (Ginebra, FIDIC).

Fournier, Jacques (1981) "Engineering in Verbo, Contrats et obligations" Recueil Dalloz de Droit Commerciel.

Frehills Smith, Herbert (2009) “The prevention principle, time at large and extension of time clauses". Disponible en: http://www.lexology.com/library/detail

García Conesa, Antonio (1996) Derecho de la Construcción (Barcelona).

García Gil, Francisco (1995): El Contrato de Ejecución de Obra y su Jurisprudencia (Madrid).

Hewitt, Andy (2011): Construction Claims \& Responses (Blackwell UK).

Huidobro Salas, Ramón (1994): "El Contrato de Construcción de Obra Pública", Revista Gaceta Jurídica No 170: pp. 20-30.

Huse, Joseph A. (2002): Understanding and Negotiating Turnkey and EPC Contracts, (Sweet and Maxwell, London).

ICe-Institute of Civil Engeineers (2001) Design and Construct Conditions (Thomas) Telford Publishing, Londres).

Ice-Institution of Civil Engineers (2011): Manual of Construction Law (London, ICE).

Illanes Ríos, Claudio (1996): El Contrato de Construcción a suma alzada, charla dictada el 27 de junio, Colegio de Abogados de Chile.

Illanes, Claudio (2005): "La Teoría de la Imprevisión en la Ley de Concesiones 
de Obras Públicas", Revista del Abogado $\mathrm{N}^{\circ} 28$.

Jenkins, Jane; Stebbings, Simon (2006): International Construction Arbitration Law (Kluwer Law Int.-USA).

Jerez Riffo, José y Palacios Baeza, Ismael (1968): Estudio Legal y Práctico sobre la Construcción (Santiago).

Jonathan Kay: FIDIC "Design and build Turnkey and EPC Contracts", The International Construction Law Review, vol. 16, No1: pp. 27-38.

María Mullerat, Ramón (1977): "La Denominación del Contrato de Construcción", Revista Jurídica de Cataluña: pp. 681 y ss.

Mira Ripolles, Mario (2001): El Contrato de Obra Pública, Reglamentación y Jurisprudencia Administrativa (Santiago).

Murdoch, John; Hughes, Will (1998): Construction Contracts (U.K, 3rd edit.).

O’Brien, James J. (1998): Construction Change Orders: Impact, Avoidance, and Documentation (McGraw-Hill USA).

Pakistan Engineering Council (2010): Standard Form Of Bidding Documents For EPC/TURNKEY Contracts (Islamabad, First Edition).

Pinnell, Steven S. (1998): How to Get Paid for Construction Changes (McGraw-Hill USA).

Podetti, Humberto (2004): Contrato de Construcción (Buenos Aires).

Prat Echaurren, Jorge (1940): Nociones sobre el Contrato de Construcción Privada, Memoria de Prueba (Santiago).

Pulido Morgan, Alberto (1962): "Los créditos refaccionarios en la ley de quiebras", Revista de Derecho y Jurisprudencia, tomo LIX, primera parte: pp. 80-109.
Rubin, Robert A., et al.: Construction Claims: Prevention and resolution (John Wiley, USA, 3rd edit.).

Sandoval López, Ricardo (2003): Contratos Mercantiles, tomo II (Santiago).

Scriven, John; Pritchard, Nigel (2011): EPC Contracts and Major Projects, A Guide to Construction and other Project Contracts (Sweet and Maxwell 2nd ed., London).

Smith, Currie \& Hancock's (2011): Common Sense Construction Law (USA, John Wiley and Sons, $4^{\text {th }}$ edition).

Stuart H. Bartholomew (1998): Construction Contracting Business and Legal Principles (Prentice Hall, USA)

Trauner, Theodore J., Jr. (1990): Construction Delays (RS Means, $1^{\text {st }}$ edit USA).

WeIl, Alex y Terré, François (1975): Droit Civil l, Les obligations, $N^{\circ} 397$ (Paris, Dalloz, Deuxieme Edit.)

WerREMEYER, Kit (2006): Understanding \& Negotiating Construction Contracts (RS Means USA).

\section{LEYES CITADAS}

Código Civil argentino

Código Civil de Bolivia

Código Civil del Perú

\section{JURISPRUDENCIA CITADA}

Sentencia arbitral, Andrés Cúneo M., 29 de julio de 2010, CAM Santiago Rol $1087-$ 2009. Art. $4 \mathrm{~N}^{\circ} 14$ del D.S. 75.

Sentencia de la Audiencia Territorial de Oviedo, España, de fecha 15 de febrero de 1978.

Sentencia de la I. Corte de Apelaciones de La Serena, de fecha 13 de enero de 2012, Rol 1670-2011.-(Civil). Casación y Apelación. 


